\title{
ADESÃO AO TRATAMENTO MEDICAMENTOSO DE PACIENTES COM DOENÇAS INFLAMATÓRIAS INTESTINAIS ACOMPANHADOS NO AMBULATÓRIO DE UM HOSPITAL UNIVERSITÁRIO
}

\author{
Nathalie de Lourdes Souza DEWULF², Rosane Aparecida MONTEIRO², Afonso Dinis Costa PASSOS², \\ Elisabeth Meloni VIEIRA² e Luiz Ernesto de Almeida TRONCON1
}

RESUMO - Racional - A adesão ao tratamento medicamentoso nas doenças inflamatórias intestinais apresenta grande importância clínica e social. Porém, são escassos os estudos sobre este tema em nosso meio. Objetivo - Investigou-se a adesão ao tratamento medicamentoso prescrito, bem como a influência de alguns fatores sobre a adesão, de pacientes com doenças inflamatórias intestinais em acompanhamento em ambulatório de Gastroenterologia de um hospital universitário ligado ao Sistema Único de Saúde (SUS). Método - Realizou-se estudo transversal, com métodos indiretos, para avaliar a adesão ao tratamento de 26 casos da doença de Crohn, 26 pacientes com retocolite ulcerativa e 4 com colite indeterminada, que faziam uso contínuo de medicamentos, dos quais $89,3 \%$ eram fornecidos pelo SUS. Os pacientes foram classificados como tendo alto ou baixo grau de adesão, com base em dois diferentes instrumentos. Resultados - A análise dos medicamentos utilizados revelou baixa adesão em 15,4\% de pacientes com doença de Crohn e 13,3\% com retocolite ulcerativa. Porém, o teste de Morisky, que avalia hábitos de uso dos medicamentos, mostrou 50\% de baixa adesão na doença de Crohn e 63,3\% na retocolite ulcerativa. Análise univariada evidenciou na doença de Crohn relação entre baixa adesão e maior duração da doença, estado marital instável, residência próxima ao hospital e envolvimento do cólon. Na retocolite ulcerativa observou-se relação entre baixa adesão e atividade da doença e maior número de medicamentos em uso. Porém, a análise multivariada não evidenciou relação estatisticamente significativa que indicasse influência de qualquer fator sobre a adesão ao tratamento. Conclusões - Proporções elevadas de pacientes com doenças inflamatórias intestinais apresentam hábitos de uso de medicamentos indicativos de baixa adesão, difíceis de prever a partir de dados demográficos e clínicos, o que aponta para a necessidade de maior atenção dos profissionais de saúde a este importante aspecto do tratamento.

DESCRITORES - Enteropatias inflamatórias, quimioterapia. Doença de Crohn, quimioterapia. Colite ulcerativa, quimioterapia. Uso de medicamentos. Cooperação do paciente. Pacientes ambulatoriais.

\section{INTRODUÇÃO}

Nas doenças crônicas cujo tratamento demanda o uso contínuo de medicamentos, para maior eficácia da terapêutica, é de estrita importância haver adesão do paciente ao regime medicamentoso prescrito. A adesão pode ser conceituada como o grau de concordância entre o comportamento de uma pessoa em relação às orientações do médico ou de outro profissional de saúde ${ }^{(13,23,34)}$. O baixo grau de adesão pode afetar negativamente a evolução clínica do paciente e a sua qualidade de vida, constituindo-se em problema relevante, que pode trazer conseqüências pessoais, sociais e econômicas ${ }^{(14)}$.

A adesão à terapêutica é um fenômeno sujeito à influência de múltiplos fatores que afetam diretamente o paciente. Os fatores que determinam o comportamento da pessoa em relação às recomendações referentes ao tratamento de sua doença estão relacionados as suas condições demográficas e sociais, à natureza da doença, às características da terapêutica, ao seu relacionamento com os profissionais de saúde, bem como a características outras, intrínsecas ao próprio paciente ${ }^{(33,34)}$.

As doenças inflamatórias intestinais (DII) que, em seu senso estrito, incluem a doença de Crohn (DC) e a retocolite ulcerativa (RCU), são afecções crônicas com incidência crescente em todo o mundo ${ }^{(6,9,28)}$. Caracterizam-se por inflamação crônica do intestino e não têm causa ainda definitivamente esclarecida ${ }^{(4,10)}$. Ainda que se admita a impossibilidade de cura para a maioria dos casos, vários tipos de tratamento medicamentoso têm sido empregados para seu controle. As DII constituem importante problema de saúde, pois atingem população relativamente jovem e

Trabalho realizado no Departamento de Clínica Médica da Faculdade de Medicina de Ribeirão Preto da Universidade de São Paulo, Ribeirão Preto, SP. Departamentos de ${ }^{1}$ Clínica Médica e ${ }^{2}$ Medicina Social da Faculdade de Medicina de Ribeirão Preto da Universidade de São Paulo, Ribeirão Preto, SP.

Correspondência: Dr. Luiz Ernesto de Almeida Troncon - Departamento de Clínica Médica - Faculdade de Medicina de Ribeirão Preto - USP Av. Bandeirantes, 3900 - 14048-900 - Ribeirão Preto, SP. E-mail: ledatron@fmrp.usp.br 
podem apresentar formas clínicas mais graves, o que implica, portanto, alto custo para a sociedade ${ }^{(4,10)}$. Soma-se a isso que os pacientes com a $\mathrm{DC}$ e a RCU, devido à necessidade freqüente de procedimentos específicos, recorrem, principalmente, ao atendimento de nível terciário ${ }^{(7)}$.

Nos ambulatórios dos hospitais universitários brasileiros são atendidos pacientes com doenças de alta complexidade, de natureza crônica e que, não raramente, demandam tratamentos prolongados. Para o tratamento das DII, os serviços públicos de assistência à saúde que estão integrados ao Sistema Único de Saúde (SUS) podem fornecer os medicamentos aos pacientes, utilizando-se de programas especiais (medicamentos essenciais ou de alto custo, de liberação excepcional), que se baseiam em protocolos clínicos e diretrizes terapêuticas bem estabelecidos ${ }^{(2,3)}$. Assim, eventual baixo grau de adesão ao tratamento ou a má utilização desses medicamentos pode, também, afetar a otimização do funcionamento do SUS e a utilização dos seus recursos.

Ainda que vários estudos tenham sido desenvolvidos com o objetivo de melhor definir os fatores de maior ou menor adesão ao tratamento medicamentoso, por parte de pacientes com doenças crônicas $^{(34)}$, são escassos os trabalhos referentes à DII. A baixa adesão de pacientes acometidos por DII foi relatada por Van HEES e Van TONGEREN ${ }^{(32)}$. Por outro lado, uma investigação em pacientes com RCU revelou índices de adesão bem superiores aos esperados ${ }^{(14)}$. Outro trabalho realizado ${ }^{(22)}$ evidenciou que a falta de adesão ao tratamento em pacientes com DII está diretamente ligada à ocorrência de distúrbios na esfera psiquiátrica, mais do que a outros fatores. Mais recentemente, outras pesquisas demonstraram alto grau de não-adesão de pacientes com DII ${ }^{(18,27,29)}$, o que pode aumentar em até 5 vezes o risco de reativação da doença ${ }^{(15)}$. Porém, ainda persiste a necessidade de realizar mais trabalhos com pacientes com DII, sobretudo no Brasil. Em achados anteriores, verificou-se que o grau de adesão ao tratamento em pacientes com DII era semelhante ao de acometidos por outras doenças digestivas e não se encontrou associação entre adesão e fornecimento do medicamento ${ }^{(5)}$.

Assim, o presente estudo teve o objetivo de investigar o grau de adesão ao tratamento medicamentoso prescrito e identificar possíveis causas associadas a eventual baixo grau de adesão, em pacientes com DII em acompanhamento no ambulatório de Gastroenterologia de um hospital universitário brasileiro ligado ao SUS.

\section{MÉTODO}

\section{Desenho do estudo e considerações éticas}

O trabalho foi realizado no Ambulatório de Gastroenterologia (Departamento de Clínica Médica) do Hospital das Clínicas da Faculdade de Medicina de Ribeirão Preto da Universidade de São Paulo (HCFMRP-USP) que, como autarquia da Secretaria de Estado da Saúde, é ligado ao SUS. Realizouse um estudo transversal, utilizando a entrevista estruturada como principal método indireto e quantitativo para avaliar o grau de adesão ao tratamento medicamentoso prescrito. $\mathrm{O}$ projeto de pesquisa foi aprovado pelo Comitê de Ética em Pesquisa da instituição (processo número 4964/2003) e se obteve o consentimento escrito, livre e esclarecido, de todos os pacientes incluídos.

\section{Pacientes}

Participaram do estudo 56 pacientes, sendo 26 com DC, 26 RCU e 4 com colite indeterminada (CI). O critério principal de inclusão foi a prescrição de uso contínuo e regular de medicamentos para o tratamento da DII, demonstrado pelo registro deste dado no prontuário médico, que era examinado com antecedência. Outros critérios de inclusão foram: idade igual ou superior a 18 anos, ter consultas regulares agendadas no período do estudo e estar, no dia da entrevista, pelo menos na segunda consulta. Foram excluídos os pacientes com deficiências que impedissem a comunicação ou que estivessem em condições clínicas que desaconselhassem sua participação no estudo.

Os pacientes foram entrevistados uma única vez, logo antes da consulta médica agendada para aquele dia, em local reservado, sendo colhidos dados referentes aos últimos 30 dias de tratamento. Todas as entrevistas foram realizadas no período de junho de 2003 a dezembro de 2004, no próprio Ambulatório de Gastroenterologia do HCFMRP-USP, sendo conduzidas pelo mesmo entrevistador.

\section{Instrumentos e critérios}

Foi utilizado um questionário estruturado e padronizado, contendo teste específico para o grau de adesão (teste de Morisky) e um formulário de análise de prontuário, desenvolvidos com base em estudos já publicados ${ }^{(13,24,33)}$. Com a aplicação desses instrumentos, obtiveram-se as variáveis demográficas, sociais e clínicas do paciente, bem como dados sobre as principais características da prescrição do tratamento medicamentoso.

Para identificar o grau de adesão ao tratamento medicamentoso, considerou-se a análise dos medicamentos utilizados e do comportamento admitido em relação ao seu uso, definido pelo teste de Morisky ${ }^{(20,21)}$. Por meio da análise dos medicamentos utilizados, foi possível verificar se os pacientes estavam tomandoos adequadamente, conforme a prescrição médica. Perguntavase ao paciente: a) se estava fazendo uso de medicamento; b) qual tomava; c) qual a dose diária em uso e d) qual a posologia empregada (o número de vezes e horário em que deveriam ser tomados). Essas informações eram, então, cotejadas com os dados registrados do prontuário referentes à consulta médica imediatamente anterior à data da entrevista e que continham a prescrição dos medicamentos. A partir dos dados obtidos neste cotejo, os pacientes foram classificados em dois grupos: baixo e alto grau de adesão.

Foram classificados como apresentando alto grau de adesão aqueles que afirmavam tomar os medicamentos prescritos e declaravam corretamente o seu nome. Foram classificados como tendo baixo grau de adesão os que relataram: a) tomar medicamentos a mais do que os prescritos; b) tomar medicamentos a menos que os prescritos; c) tomar outros medicamentos ao invés dos prescritos e d) não tomar os medicamentos prescritos para o tratamento. Ressalte-se que esta análise foi realizada tendo como referência exclusivamente o tratamento medicamentoso específico para a doença inflamatória intestinal.

O teste de Morisky ${ }^{(20,21)}$ é composto por quatro perguntas, que objetivam avaliar o comportamento do paciente em relação ao uso habitual do medicamento (Figura 1). O paciente foi classificado no grupo de alto grau de adesão, quando as 
respostas a todas as perguntas foram negativas. Porém, quando pelo menos uma das respostas foi afirmativa, o paciente foi classificado no grupo de baixo grau de adesão. Essa avaliação permite, também, discriminar se o comportamento de baixo grau de adesão é do tipo intencional ou não-intencional, sendo também, possível caracterizar pacientes acometidos por ambos os tipos de comportamento de baixa adesão(27).

\begin{tabular}{|lcc|}
\hline Perguntas referentes ao teste de Morisky & $\begin{array}{c}\text { Não } \\
\text { intencional }\end{array}$ & Intencional \\
\hline $\begin{array}{l}\text { Você, alguma vez, esquece de tomar o seu remédio? } \\
\text { Você, às vezes, é descuidado }\end{array}$ & $\mathrm{X}$ & \\
$\begin{array}{l}\text { quanto ao horário de tomar o seu remédio? } \\
\text { Quando você se sente bem, } \\
\text { alguma vez, você deixa de tomar seu remédio? }\end{array}$ & $\mathrm{X}$ & $\mathrm{X}$ \\
$\begin{array}{l}\text { Quando você se sente mal com } \\
\text { o remédio, às vezes, deixa de tomá-lo? }\end{array}$ & $\mathrm{X}$ \\
\hline
\end{tabular}

FIGURA 1. Perguntas que compõe o teste de Morisky e classificação dos tipos de comportamento de baixo grau de adesão, indicado por resposta afirmativa a pelo menos uma delas ${ }^{(27)}$

Como variáveis independentes, foram estudados 16 possíveis fatores relacionados ao grau de adesão. Esses dados foram classificados em diferentes grupos, considerando os dados demográficos, sociais, clínicos e os relacionados ao tratamento medicamentoso.

Para se definir o diagnóstico principal e as características das DII, quando do preenchimento do formulário de análise do prontuário, consideraram-se as anotações dos diagnósticos médicos registrados explicitamente, os quais por sua vez, eram baseados no quadro clínico e nos resultados de exames endoscópicos, radiológicos, histológicos e laboratoriais de cada caso. Em relação à extensão e a localização da doença, na DC consideraram-se as seguintes possibilidades: íleo, íleo-cólica, somente no cólon e outros segmentos do tubo digestivo $^{(19)}$. No caso da RCU, considerou-se essa variável definida por proctite, retossigmoidite, colite esquerda, ou colite extensa ${ }^{(8)}$. Em relação à forma da doença, na $\mathrm{DC}$, consideraram-se as seguintes possibilidades: inflamatória, estenosante e fistulisante ${ }^{(19)}$. Nos casos da RCU, a variável forma foi considerada como leve/transitória, moderada ou grave $^{(30)}$. Em relação à atividade da doença, tanto para a DC como para a RCU, considerou-se a doença ativa, em remissão ou indeterminada. Para isto, utilizaram-se registros explícitos no prontuário do diagnóstico médico ou quando ausentes, a análise retrospectiva de dados clínicos, de exames laboratoriais e da conduta terapêutica, que foram interpretados tendo como referência índices de atividade usuais para a $\mathrm{DC}^{(12)}$ e para a $\mathrm{RCU}^{(31)}$.

\section{Análise de dados}

Os dados colhidos na entrevista e no exame do prontuário foram armazenados em banco de dados específico, criado no ambiente do programa estatístico Epi-info ${ }^{\mathrm{TM}}$, versão 3.2 Windows ${ }^{\circledR}$, NT, criado pelo CDC (Centers for Disease Control and Prevetion) de domínio público (disponível em http://www. cdc.gov/epiinfo). Como forma de minimizar erros, a entrada dos dados no programa foi realizada com dupla digitação.
Possíveis associações entre baixa adesão ao tratamento medicamentoso e variáveis diversas, como: escolaridade, estado marital, procedência, ocupação, extensão e localização, forma da doença, atividade da doença, modo de aquisição dos medicamentos e número de medicamentos, foram inicialmente verificadas mediante o teste exato de Fisher. Os casos com colite indeterminada foram integrados ao grupo de pacientes com RCU, dada à semelhança clínica entre as entidades. $\mathrm{Na}$ análise das diversas variáveis, os sujeitos foram classificados em duas categorias, da seguinte maneira: escolaridade (até ensino fundamental completo versus níveis superiores); estado marital (com parceiro versus sem parceiro); procedência (residentes em Ribeirão Preto versus residentes em outros municípios); ocupação (com renda própria versus dependentes); extensão e localização (na DC: envolvimento do cólon versus não envolvimento; na RCU: colite extensa versus outras formas); forma da doença (na DC: inflamatória versus outras; na RCU: leve/transitória versus outras); atividade da doença (em atividade versus remissão ou indeterminada); aquisição da medicação (fornecimento integral pelo SUS versus aquisição pelo paciente); número de medicamentos em uso (apenas um versus quantidades maiores). As variáveis que, na análise preliminar pelo teste de Fisher apresentaram $P \leq 0,20$, foram incluídas num modelo de regressão logística não condicional, testando-se previamente para a existência de interações. Em todas as situações, o limite de significância estatística foi fixado em $P<0,05$. Nas várias análises, utilizaramse os programas Epi-info e Stata 6.0 ${ }^{\mathrm{TM}}$.

\section{RESULTADOS}

\section{Características dos pacientes}

As características demográficas dos pacientes incluídos no estudo são apresentadas na Tabela 1 . Houve predomínio de mulheres com idade abaixo de 40 anos dentre os doentes com DC e de homens com idade acima de 40 anos no grupo com RCU. Ambos os grupos apresentaram predomínio de pacientes com parceiros e com no máximo o ensino fundamental, procedentes de outra cidade do Estado de São Paulo.

TABELA 1 - Características demográficas dos pacientes incluídos no estudo

\begin{tabular}{lcc}
\hline Características & $\begin{array}{c}\text { DC } \\
(\mathbf{n}=26)\end{array}$ & $\begin{array}{c}\text { RCU } \\
(\mathbf{n}=30)\end{array}$ \\
\hline $\begin{array}{l}\text { Idade } \\
\quad \text { Acima de } 40 \text { anos }\end{array}$ & $9(34,6)$ & $17(56,7)$ \\
Sexo $\quad$ & $10(38,5)$ & $22(73,3)$ \\
$\quad$ Homens & & \\
Estado marital & $18(69,2)$ & $20(66,6)$ \\
$\quad$ Com parceiro & $16(61,5)$ & $21(70,0)$ \\
Escolaridade & & \\
$\quad$ Até ensino fundamental completo & $5(19,2)$ & $9(30,0)$ \\
Procedência & $17(65,4)$ & $18(60,0)$ \\
$\quad \begin{array}{l}\text { Ribeirão Preto } \\
\quad \text { Procedente de outras cidades do Estado de SP }\end{array}$ & $4(15,4)$ & $3(10,0)$ \\
$\quad$ Procedentes do outros Estados & & \\
\hline DC: pacientes com doença de Crohn; RCU: pacientes com retocolite ulcerativa &
\end{tabular}

$\mathrm{n}=$ número absoluto de pacientes em cada grupo; ( ) percentuais. 
A Tabela 2 apresenta algumas das características sociais dos pacientes estudados. A maioria possuía fonte de renda própria, com predomínio de trabalhadores autônomos. Houve predomínio de rendimentos acima de um salário mínimo na DC e de até um salário mínimo na RCU. A maioria dos pacientes de ambos os grupos relatou que os medicamentos eram fornecidos integralmente pelo SUS.

TABELA 2. Características sociais dos pacientes incluídos no estudo

\begin{tabular}{lcc}
\hline Características & $\begin{array}{c}\text { DC } \\
(\mathbf{n}=26)\end{array}$ & $\begin{array}{c}\text { RCU } \\
(\mathbf{n}=30)\end{array}$ \\
\hline $\begin{array}{l}\text { Ocupação } \\
\quad \text { Com renda própria }\end{array}$ & $18(69,2)$ & $23(76,6)$ \\
Renda per capita & & \\
$\quad$ Até um salário mínimo & $10(38,5)$ & $15(50,0)$ \\
$\quad \begin{array}{l}\text { Acima de um salário } \\
\text { Sem relato }\end{array}$ & $16(61,5)$ & $12(40,0)$ \\
Aquisição dos medicamentos & - & $3(10,0)$ \\
$\quad \begin{array}{l}\text { Fornecido integralmente pelo SUS } \\
\text { Adquirido pelo paciente: }\end{array}$ & $24(92,3)$ & $26(86,7)$ \\
$\quad \begin{array}{l}\text { Totalmente } \\
\quad \text { Parcialmente }\end{array}$ & $2(7,7)$ & $2(6,7)$ \\
\end{tabular}

DC: pacientes com doença de Crohn; RCU: pacientes com retocolite ulcerativa

SUS: Sistema Único de Saúde; $\mathrm{n}$ = número absoluto de pacientes em cada grupo; () percentuais

Quanto às características clínicas, em $61,5 \%$ dos pacientes com DC, o tempo de doença era menor de 5 anos e $61,5 \%$ dos casos eram acometidos por outras doenças. Metade destes pacientes havia sofrido operações prévias e 53,8\% necessitaram de ao menos uma internação relacionada à DC. Neste grupo, houve predomínio da localização íleo-cólica (Figura 2), da forma penetrante (Figura 3) e de casos em remissão (Figura 4), quando da inclusão no estudo. Nos pacientes com RCU, o tempo de doença era maior que 5 anos em 53,3\% dos casos, $60 \%$ deles eram acometidos por outras doenças, $26,7 \%$ necessitaram de

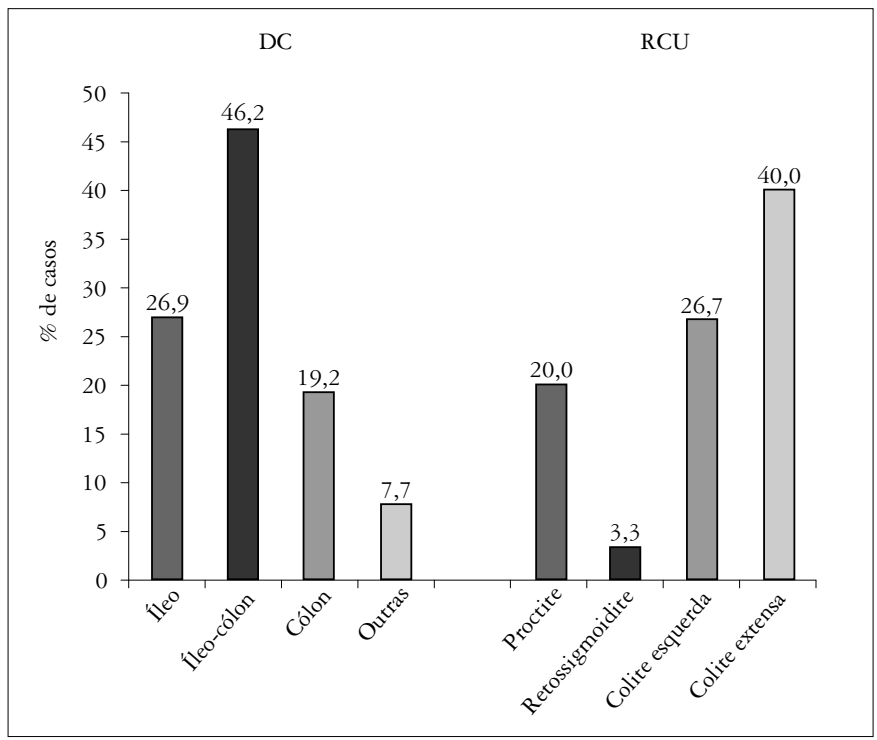

FIGURA 2. Distribuição percentual dos casos da doença de Crohn (DC) e retocolite ulcerativa (RCU) quanto à localização e extensão predominante das lesões. Outras: duodeno distal e jejuno proximal ao menos uma internação e apenas 3,3\% dos pacientes haviam necessitado de operações. Na RCU, houve predomínio da colite extensa (Figura 2), da forma leve ou transitória (Figura 3) e de casos em remissão (Figura 4).

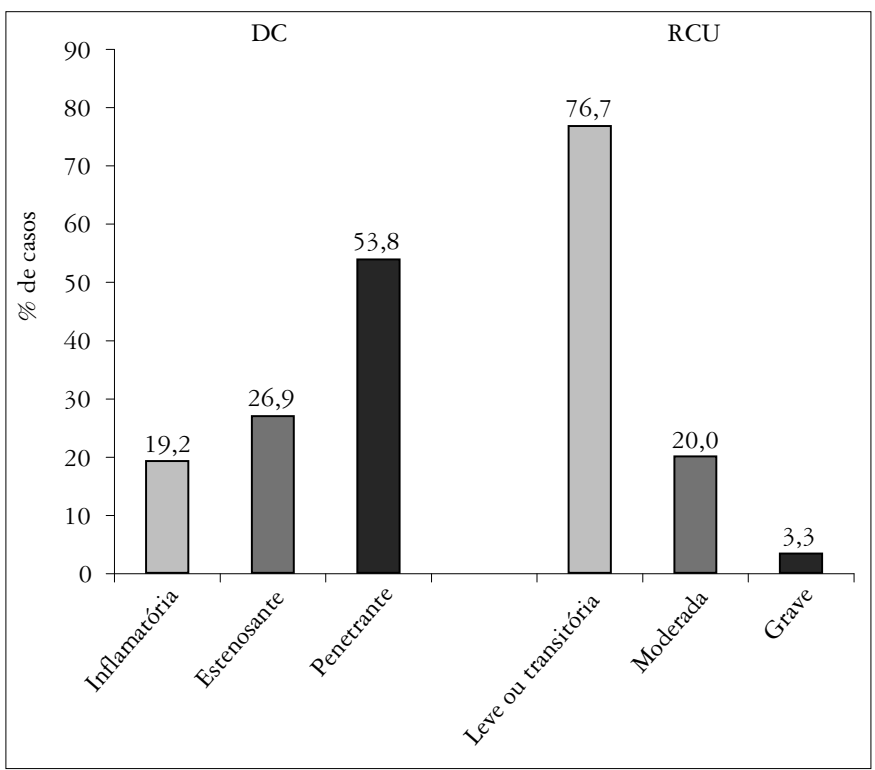

FIGURA 3. Distribuição percentual dos casos da doença de Crohn (DC) e retocolite ulcerativa (RCU) quanto à forma

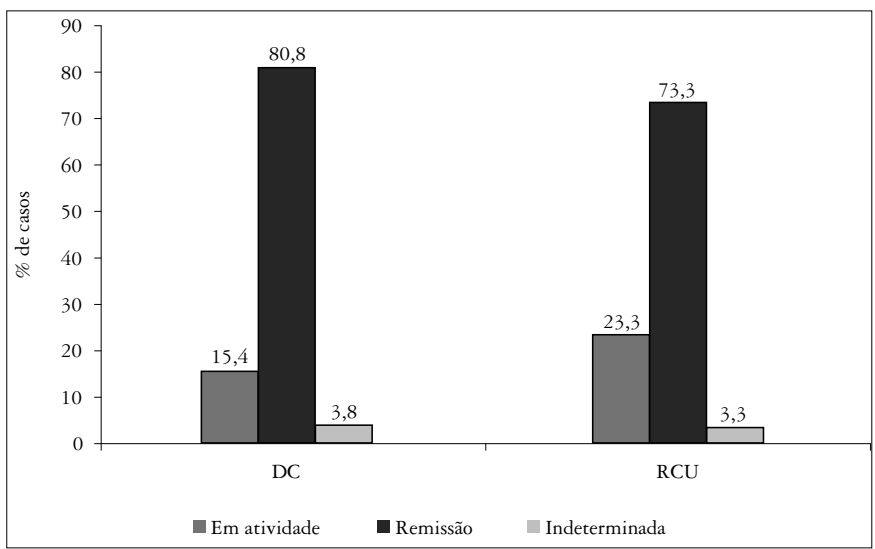

FIGURA 4. Distribuição percentual dos casos da doença de Crohn (DC) e retocolite ulcerativa (RCU) quanto à atividade da doença no momento da inclusão no estudo

Quanto às características do tratamento medicamentoso prescrito especificamente para as DII, houve predomínio do uso de apenas um medicamento (Tabela 3). Os medicamentos mais utilizados (Tabela 4) foram azatioprina para os pacientes com DC e aminossalicilatos para o tratamento da RCU. Os aminossalicilatos utilizados foram sulfassalazina e mesalazina, em comprimidos ou supositórios. Os corticosteróides utilizados eram prednisona, em comprimidos, e hidrocortisona, na forma de enema. Os antibacterianos, metronidazol ou ciprofloxacina, eram utilizados por via oral. 
TABELA 3. Número de medicamentos em uso por parte dos 56 pacientes incluídos no estudo

\begin{tabular}{lcc}
\hline Número & $\begin{array}{c}\text { DC } \\
(\mathbf{n}=26)\end{array}$ & $\begin{array}{c}\text { RCU } \\
(\mathbf{n}=30)\end{array}$ \\
\hline Um & $16(61,5)$ & $17(56,7)$ \\
Dois & $7(26,9)$ & $9(30,0)$ \\
Três & $2(7,7)$ & $4(13,3)$ \\
Quatro & $1(3,8)$ & 0 \\
\hline
\end{tabular}

DC: pacientes com doença de Crohn; RCU: pacientes com retocolite ulcerativa

$\mathrm{n}$ : número absoluto de pacientes em cada grupo () percentuais

TABELA 4. Dados sobre a classe terapêutica dos medicamentos em uso pelos pacientes entrevistados, com doença inflamatória intestinal

\begin{tabular}{lcc}
\hline Classe & $\begin{array}{c}\text { DC } \\
(\mathbf{n}=26)\end{array}$ & $\begin{array}{c}\text { RCU } \\
(\mathbf{n}=30)\end{array}$ \\
\hline Aminossalicilatos & $2(7,7)$ & $20(66,8)$ \\
Azatioprina & $11(42,4)$ & $1(3,3)$ \\
Corticosteróides & $2(7,7)$ & - \\
Metronidazol ou ciprofloxacina & $1(3,8)$ & - \\
Aminossalicilatos e azatioprina & $1(3,8)$ & $4(13,3)$ \\
Aminossalicilatos e corticosteróides & $1(3,8)$ & $4(13,3)$ \\
Azatioprina e corticosteróides & $4(15,4)$ & $1(3,3)$ \\
Associaç̃̃es de três ou mais medicamentos & $4(15,4)$ & - \\
\hline
\end{tabular}

DC: pacientes com doença de Crohn; RCU: pacientes com retocolite ulcerativa

$\mathrm{n}$ : número absoluto de pacientes em cada grupo; () percentuais

\section{Adesão ao tratamento medicamentoso}

As proporções de pacientes classificados como apresentando baixo ou alto grau de adesão ao tratamento medicamentoso prescrito são apresentadas na Tabela 5. A análise dos medicamentos utilizados revelou que apenas quatro pacientes $(15,4 \%)$ com DC e quatro $(13,3 \%)$ com RCU foram classificados em baixa adesão. Dentre os quatro casos com DC em que foi identificada a baixa adesão, dois $(7,7 \%)$ tomavam medicamentos a menos que os prescritos e dois $(7,7 \%)$ não os tomavam. Dentre os quatro casos de pacientes com RCU e com baixa adesão, dois $(6,6 \%)$ tomavam medicamentos a mais e dois $(6,6 \%)$ tomavam medicamentos a menos que os prescritos.

Este perfil de adesão foi alterado drasticamente quando se avaliou o comportamento do uso habitual do(s) medicamento(s) pelo teste de Morisky (Tabela 5). De fato, observaram-se nos dois grupos, percentagens mais elevadas de casos sendo classificados como de baixo grau de adesão: $50 \%$ dos pacientes com DC e

TABELA 5. Dados sobre adesão ao tratamento medicamentoso prescrito dos pacientes incluídos no estudo, referente à análise dos medicamentos utilizados e ao teste de Morisky

\begin{tabular}{|c|c|c|c|c|}
\hline \multirow{2}{*}{ Grau de adesão } & \multicolumn{2}{|c|}{$\begin{array}{c}\text { Analise dos } \\
\text { medicamentos utilizados }\end{array}$} & \multicolumn{2}{|c|}{ Teste de Morisky ${ }^{(20,21)}$} \\
\hline & $\begin{array}{c}\text { DC } \\
(n=26)\end{array}$ & $\begin{array}{c}\mathrm{RCU} \\
(\mathrm{n}=30)\end{array}$ & $\begin{array}{c}\text { DC } \\
(n=26)\end{array}$ & $\begin{array}{c}\mathrm{RCU} \\
(\mathrm{n}=30)\end{array}$ \\
\hline Alto grau de adesão & $22(84,6)$ & $26(86,7)$ & $13(50,0)$ & $11(36,7)$ \\
\hline Baixo grau de adesão & $4(15,4)$ & $4(13,3)$ & $13(50,0)$ & $19(63,3)$ \\
\hline Não intencional & - & - & $11(42,3)$ & $18(60,0)$ \\
\hline Intencional & - & - & - & - \\
\hline $\begin{array}{l}\text { Ambos os tipos de } \\
\text { comportamento }\end{array}$ & - & - & $2(7,7)$ & $1(3,3)$ \\
\hline Total & 26 & 30 & 26 & 30 \\
\hline
\end{tabular}

DC: pacientes com doença de Crohn; RCU: pacientes com retocolite ulcerativa

$\mathrm{n}=$ número absoluto de pacientes em cada grupo; ( ) percentuais
$63,3 \%$ com RCU. Entre esses pacientes, a maioria apresentava comportamento de baixa adesão do tipo não intencional $(42,3 \%$ na DC e $60 \%$ na RCU), indicando padrões de esquecimento ou de descuido com o horário para tomar o medicamento. Ainda dentre os pacientes classificados como tendo baixa adesão, alguns poucos apresentaram ambos os tipos de comportamento (7,7\% na DC e 3,3\% na RCU). Destaque-se que nenhum caso foi classificado como baixa adesão do tipo intencional.

\section{Fatores que afetam à adesão}

Não houve diferença entre a DC e a RCU quanto ao percentual de casos classificados como menos aderentes, tanto na análise dos medicamentos utilizados $(15,4 \%$ versus $13,3 \% ; P=0,56)$, como nos resultados do teste de Morisky $(50,0 \%$ versus $63,3 \% ; P=0,23)$.

No grupo de pacientes com DC, verificou-se proporção significativamente maior $(P=0,01)$ de pacientes com até 5 anos de duração da doença, dentre os classificados como mais aderentes. Na RCU, houve proporção significantemente maior $(P=0,03)$ de pacientes em que a doença estava em remissão ou em atividade indeterminada, entre os classificados como mais aderentes. Neste grupo com RCU, houve, também, proporção significantemente maior $(P=0,03)$ de casos classificados como mais aderentes, dentre aqueles que utilizavam somente um medicamento.

$\mathrm{Na}$ análise univariada dos fatores que influenciam o grau de adesão ao tratamento medicamentoso, definido pelo teste de Morisky, verificou-se que na DC houve associação estatisticamente significante $(P=0,01)$ entre maior grau de adesão ao tratamento e o estado marital estável e a procedência de outra cidade, que não a de Ribeirão Preto. Além disso, observou-se associação estatisticamente significante $(P=0,04)$ entre envolvimento do cólon e menor grau de adesão ao tratamento na DC.

A análise multivariada, porém, mostrou que nenhuma das variáveis demográficas, sociais, clínicas ou da terapêutica, apresentou associação estatisticamente significante com o grau de adesão ao tratamento prescrito

\section{DISCUSSÃO}

Neste estudo, avaliando-se o uso do medicamento associado ao conhecimento do seu nome pelos pacientes com DII, verificou-se menor adesão ao tratamento em proporções relativamente baixas dos casos (15,4\% na DC e 13,3\% na RCU). Porém, utilizando o teste de Morisky, observou-se que metade ou mais dos pacientes apresentou comportamento indicativo de baixo grau de adesão (50,0\% na DC e 63,8\% na RCU), com predominância do tipo não intencional (descuido, esquecimento). Estes dados indicam alto grau de disposição para o uso do medicamento, mas baixo grau de preocupação dos pacientes com a sua utilização correta.

A adesão do paciente ao tratamento tem sido avaliada por métodos diretos e indiretos. Os primeiros permitem detectar os medicamentos ou produtos da sua metabolização nos fluidos biológicos do paciente ${ }^{(13,33)}$, enquanto os indiretos incluem entrevistas, informações obtidas de profissionais de saúde e familiares, resultados dos tratamentos ou atividades de prevenção, preenchimento de prescrições e contagem de 
comprimidos ${ }^{(13,33)}$. Neste estudo, utilizou-se a entrevista, que é um método indireto, mas de fácil aplicação, tem boa aceitação pelos participantes e apresenta custo reduzido ${ }^{(13)}$. Assim, na análise dos dados obtidos com a entrevista, devem-se levar em consideração as limitações intrínsecas a este método. Em trabalhos em que se compararam os índices de adesão por métodos diretos e indiretos, observou-se que o paciente verdadeiramente aderente dificilmente informa não-adesão ao tratamento ${ }^{(13)}$. Do mesmo modo, o paciente que se declara não aderente, provavelmente, está descrevendo a verdade ${ }^{(13)}$. Porém, muitos pacientes relatam o uso correto do medicamento, apenas por ser este o comportamento esperado ${ }^{(13,23)}$. Por isso, a vulnerabilidade dos métodos indiretos associa-se a maior probabilidade de haver a superestimação da adesão e, portanto, de subestimação da não-adesão ${ }^{(13,17,33)}$.

O teste de Morisky ${ }^{(20)}$, apesar de ser também indireto, possui diferentemente da entrevista, a característica de superestimar a não-adesão, devido à rigidez dos seus critérios para caracterizar o paciente como alto grau de adesão ${ }^{(17)}$. Isso fica mais evidente quando se observa que, em outros estudos, com métodos diversos, a adesão se caracteriza quando há a utilização de $80 \%$ ou mais da prescrição médica ${ }^{(15,23,29)}$.

Vários estudos indicam que a média de não-adesão ao tratamento medicamentoso, em condições clínicas diversas, é de cerca de $50 \%$, variando de $0 \%$ a $100 \%$ dos pacientes ${ }^{(23)}$. A adesão ao tratamento na DII tem sido relatada como variando de $12 \%$ a $72 \%(14,18,22,25,27,29,32)$. No presente estudo, com base nas informações sobre os medicamentos utilizados, o baixo grau de adesão apresentou-se com menor freqüência $(15,4 \%$ na DC e 13,3\% na RCU). Já com o teste de Morisky, os níveis foram mais altos, em relação a outros estudos $(50 \%$ na DC e $63,3 \%$ na $\mathrm{RCU})$ porém, concordantes com os de outros trabalhos $(41 \% \mathrm{e}$ $72 \%)$ que utilizaram o mesmo teste ${ }^{(18,27)}$.

Os baixos níveis de não-adesão observados neste estudo, a partir das informações dos pacientes sobre os medicamentos utilizados, discordam dos estudos antes relatados, o que sugere ter havido superestimação do grau de adesão. Isto pode ser devido não só ao método empregado, mas também aos critérios estabelecidos, que envolveram somente a afirmativa do uso e o conhecimento do nome do medicamento. Caso os critérios incluíssem não só o conhecimento correto do nome, mas também saber dose e posologia, é plausível que a proporção de pacientes considerados menos aderentes tivesse sido maior. Por outro lado, os altos índices de pacientes em remissão clínica da DII encontrados no presente estudo ( $80,8 \%$ na DC e $73,3 \%$ na RCU) sugerem que os medicamentos estão sendo, de fato, utilizados, ainda que não se possa descartar a possibilidade de que estejam em remissão espontânea.

O teste de Morisky mostrou que o comportamento predominante de não-adesão foi do tipo não intencional (esquecimento, descuido). $\mathrm{O}$ esquecimento, em particular, tem sido relatado como fator mais freqüente de baixo grau de adesão ao tratamento medicamentoso prescrito ${ }^{(15,18,27)}$.

A análise univariada dos possíveis fatores relacionados à baixa adesão, por meio da análise dos medicamentos utilizados, mostrou que a menor adesão do paciente com DC ao tratamento associa-se a maior duração da doença, o que contraria os achados de estudos prévios ${ }^{(22,27)}$. Já na RCU, houve associação entre menor adesão e atividade da doença, o que condiz com achados de outro estudo ${ }^{(27)}$, embora tenha sido já relatado que a nãoadesão ocorre, principalmente, em momentos de remissão ${ }^{(25)}$. Ainda na RCU, observou-se maior adesão dos pacientes que faziam uso de apenas um medicamento, o que concorda com outros relatos e é facilmente explicado pela facilidade de se lidar com menor número de produtos ${ }^{(14,29,34)}$. Quando avaliado pelo teste de Morisky, na DC, o comportamento de maior adesão mostrou relação com o estado marital estável, o que condiz com o já descrito ${ }^{(33)}$. Ainda na DC, outro fator relacionado a menor adesão foi o envolvimento do cólon, evento para o qual não se tem explicação, e pacientes procedentes de Ribeirão Preto, o que contradiz outros achados da literatura ${ }^{(34)}$. Porém, a análise multivariada não confirmou estes achados e não evidenciou um único fator que tivesse associação estatisticamente significante com a adesão ao tratamento e que fosse comum aos grupos de pacientes com DC e RCU, o que pode se dever ao pequeno tamanho dos subgrupos analisados.

A ausência de diferença entre os achados na DC e na RCU, bem como a semelhança entre a DII e outras doenças digestivas $^{(5)}$, sugere um padrão de comportamento comum aos usuários do serviço, independentemente da doença e de outras variáveis de natureza demográfica, socioeconômica ou clínica. Esta hipótese poderia ser testada com a utilização de outras abordagens de estudo, como as que envolvem métodos da pesquisa qualitativa, que permitem aprofundar o conhecimento do tema em determinado grupo, desvendando suas outras características, de natureza mais subjetiva ${ }^{(17)}$.

Um fator que pode estar relacionado a menor adesão é a receptividade das informações, uma vez que a gravidade da condição e a intensidade dos sintomas influenciam a receptividade das informações e, conseqüentemente, afetam a intenção de seguimento do tratamento ${ }^{(11)}$. Adicionalmente, tem sido descrito que a não-adesão dos pacientes ao tratamento pode estar relacionada a três fatores principais: a) a dificuldade do médico em se comunicar com o paciente; b) a dificuldade do paciente em interpretar as informações sobre o uso do medicamento e c) a dificuldade do paciente em se organizar em casa para a administração do medicamento ${ }^{(16,26)}$. Assim, destaca-se a importância da boa comunicação do médico e dos profissionais de saúde com o paciente, visando transformar as informações por ele recebidas em conhecimentos definidos, uma vez que a falta de conhecimento ou a existência de dúvidas parecem ser, também, fatores de menor adesão( ${ }^{(24)}$. Neste sentido, na interpretação dos dados do presente estudo, deve-se também considerar o contexto do atendimento, uma vez que em hospitais universitários é alta a rotatividade na equipe médica, o que pode afetar a qualidade do vínculo entre o paciente e o médico, fator importante para a existência da confiança por parte do paciente. Esta confiança que o paciente deposita em toda a equipe de saúde, no médico responsável pela prescrição, bem como no farmacêutico que orienta seu uso correto, no próprio medicamento prescrito e no tratamento como um todo, é um possível fator de influência sobre a adesão ao tratamento ${ }^{(1,24)}$. 


\section{CONCLUSÕES}

Pacientes com DII (DC e RCU), atendidos em ambulatório especializado de hospital terciário, apresentam alto grau de adesão ao tratamento medicamentoso. Porém, a aplicação do teste de Morisky, que avalia o hábito em relação ao medicamento, mostrou que metade ou mais dos casos exibe comportamento de baixa adesão, do tipo não intencional (descuido ou esquecimento). A menor adesão ao tratamento foi semelhante na DC e na RCU e, em análise multivariada, não se associou a nenhum fator de ordem demográfica ou clínica, podendo tratar-se de traço comum aos usuários do serviço. Estes achados indicam a necessidade de medidas educacionais específicas e maior atenção dos profissionais da saúde para o uso dos medicamentos pelos pacientes.

Dewulf NLS, Monteiro RA, Passos ADC, Vieira EM, Troncon LEA. Compliance to drug therapy in inflammatory bowel diseases outpatients from an university hospital. Arq Gastroenterol. 2007;44(3):289-96.

ABSTRACT - Background - Compliance to drug therapy is important for a successful treatment. Although many studies have assessed compliance to treatment in patients with chronic diseases, few investigations have been carried out in inflammatory bowel diseases. Aims - To assess compliance to drug therapy in patients with inflammatory bowel diseases - Crohn's disease and ulcerative colitis -, followed at a university hospital, who had prescribed medication supplied by the Brazilian National Health System. Methods - In a cross sectional study, a structured interview was applied to assess the compliance of 26 Crohn's disease patients, 26 ulcerative colitis patients and 4 cases with undetermined colitis. Patients were characterized as presenting higher or lower degree of compliance, based on the comparison of the information provided by the patient in the interview and data in the medical records. The Morisky test was also used to assess the behavioral pattern of the patient regarding the daily use of the medication. Results - The interview showed that $15.4 \%$ of patients with Crohn's disease and $13.3 \%$ of those with ulcerative colitis could be regarded as less compliant. However, the Morisky test revealed lower compliance in $50 \%$ of patients with Crohn's disease and $63.3 \%$ of those with ulcerative colitis. Univariate analysis showed an association between low compliance and long disease duration, married status and colon involvement in Crohn's disease, and between low compliance and increased disease activity and greater number of medications in ulcerative colitis. However, multivariate analysis did not confirm any association between low compliance and any demographic or clinical factor. Conclusions - A high degree of noncompliance to treatment, linked to habitual behavior and hard to predict from demographic or clinical factor, was detected in inflammatory bowel disease patients, which suggests the need for investment in patient education regarding medication use.

HEADINGS - Inflammatory bowel diseases, drug therapy. Crohn disease, drug therapy. Colitis, ulcerative, drug therapy. Drugs utilization. Patient compliance. Outpatients. 


\section{REFERÊNCIAS}

1. Balkrishanan R. The importance of medication adherence in improving chronicdisease related outcomes: what we know and what we need to further know. Med Care. 2005;43:517-20.

2. Brasil. Secretaria de Assistência à Saúde. Ministério da Saúde. Protocolo clínico e diretrizes terapêuticas para o tratamento de doença de Crohn. Portaria n. 858 , 5 novembro 2002. Diário Oficial da União, Brasília (DF). 2002 nov 12; 214 Seção I:77-82.

3. Brasil. Secretaria de Assistência à Saúde. Ministério da Saúde. Protocolo clínico e diretrizes terapêuticas para o tratamento de retocolite ulcerativa. Portaria n. 861/02, 5 novembro 2002. Diário Oficial da União, Brasília (DF). 2002 nov 12;214 Seção I:87-9.

4. Carter MJ, Lobo AJ, Travis SP. Guidelines for the management of inflammatory bowe disease in adults. Gut. 2004;53 (suppl V):v1-v16.

5. Dewulf NL. Investigação sobre a adesão ao tratamento medicamentoso em pacientes com doenças inflamatórias intestinais [dissertação]. Ribeirão Preto, SP: Faculdade de Medicina de Ribeirão Preto da Universidade de São Paulo; 2005.

6. Delco F, Sonnenberg A. Commonalities in the time trends of Crohn's disease and ulcerative colitis. Am J Gastroenterol. 1999;94:2171-6.

7. Faria LC, Ferrari ML, Cunha AS. Aspectos clínicos da doença de Crohn em um centro de referência para doenças intestinais. Gastroenterol Endosc Dig. 2004;23:151-64.

8. Farmer RG. Nonspecific ulcerative proctitis. Gastroenterol Clin North Am. 1987; $16: 154-74$

9. Gaburri PD, Chebli JM, Castro LE, Ferreira JO, Lopes MH, Ribeiro AM, Alves RA, Froede EC, Oliveira KS, Gaburri AK, Gaburri D, Meirellers GS, Souza AF. Epidemiologia, aspectos clínicos e evolutivos da doença de Cronh: um estudo de 60 casos. Arq Gastroenterol. 1998;35:240-6.

10. Glickman RM. Doença inflamatória intestinal. In: Isselbacher KJ, Braunwald E, Wilson JD, Martin JB, Fauci AS, Kasper DL. Harrison. Princípios de medicina interna 13a ed. México: Nueva editorial; 1995. p.1472-85.

11. Goldring AB, Taylor SE, Kemeny ME, Anton PA. Impact of health beliefs, quality of life, and the physician-patient relationship on the treatment intentions of inflammatory bowel disease patients. Health Psychol. 2002;21:219-28.

12. Harvey RF, Bradshaw JM. A simple index of Crohn's disease activity. Lancet $1980 ; 1 \cdot 514$

13. Haynes RB, Taylor DW, Sackett DL. Compliance in health care. Baltimore, Maryland: The Johns Hopkins University Press; 1981.

14. Kane SV, Cohen RD, Aikens JE, Hanauer SB. Prevalence of nonadherence with maintenance mesalamine in quiescent ulcerative colitis. Am J Gastroenterol. 2001;96:2929-33

15. Kane S, Huo D, Aikens J, Hanauer S. Medication nonadherence and the outcomes of patients with quiescent ulcerative colitis. Am J Med. 2003;114:39-43.

16. Kane SV. Systematic review: adherence issues in the treatment of ulcerative colitis. Aliment Pharmacol Ther. 2006;23:577-85.

17. Leite SN, Vasconcelos MP. Adesão à terapêutica medicamentosa: elementos para discussão de conceitos e pressupostos adotados na literatura. Ciênc Saúde Coletiva. $2003 ; 8: 775-82$
18. López San Román A, Bermejo F, Carrera E, Pérez-Abad M, Boixeda D. Adhesión al tratamiento em la enfermedad inflamatória intestinal. Rev Esp Enferm Dig. 2005;97:249-57.

19. Louis E, Collard A, Oger AF, Degroote E, Aboul Nasr El Yafi FA, Belaiche J. Behaviour of Crohn's disease according to the Vienna classification: changing pattern over the course of the disease. Gut 2001;49:777-82.

20. Morisky DE, Levine M, Green LW, Smith CR. Health education program effects on the management of hypertension in the elderly. Arch Intern Med. 1982;142:1835-8

21. Morisky DE, Green LW, Levine DM Concurrent and predective validity of selfreported measure of medication adherence. Med Care. 1986;24:67-74.

22. Nigro G, Angelini G, Grosso SB, Caula G, Sategna-Guidetti C. Psychatric predictors of non compliance in inflammatory bowel disease. J Clin Gastroenterol. 2001;32:61-8.

23. Ostenberg L, Blaschke T. Adherence to medication. N Engl J Med. 2005;353:487-97.

24. Pepe VL, Castro CG. A interação entre prescritores, dispensadores e pacientes: informação compartilhada como possível benefício terapêutico. Cad Saúde Pública. 2000; $16: 815-22$.

25. Robinson A. Review article: inflammatory bowel disease - empowering the patient and improving outcome. Aliment Pharmacol Ther. 2004;20 (suppl 4):84-7.

26. Rogers A, Kennedy A, Nelson E, Robinson A. Uncovering the limits of patientcenteredness: implementing a self-management trial for chronic illness. Qual Health Res. 2005;15:224-39.

27. Sewitch MJ, Abrahamowicz M, Burkun A, Bitton A, Wild GE, Cohen A, Dobkin PL. Patient nonadherence to medication in inflammatory bowel disease. Am J Gastroenterol. 2003;98:1535-44

28. Souza MH, Troncon LE, Rodrigues CM, Viana CF, Onofre PH, Monteiro RA, Passo AD, Martinelli AL, Meneghelli UG. Evolução da ocorrência (1980-1999) da doença de Crohn e da retocolite ulcerativa idiopática e análise das suas características clínicas em um hospital universitário do sudeste do Brasil. Arq Gastroenterol. 2002;39:98-105.

29. Shale MJ, Riley SA. Studies of compliance with delayed-release mesalazine therapy in patients with inflammatory bowel disease. Aliment Pharmacol Ther 2003; 18:191-8

30. Stonnington CM, Phillips SF, Melton III LJ, Zinsmeister AR. Chronic ulcerative colitis: incidence and prevalence in a community. Gut. 1987;28:402-9.

31. Truelove SC, Witts LJ. Cortisone in ulcerative colitis: final report on a therapeutic trial. Br Med J. 1955;2:1041-8.

32. Van Hees PA, Van Tongeren JH. Compliance to therapy in patients on a maintenance dose of sulfasalazine. J Clin Gastroenterol. 1982;4:333-6.

33. Vermeire E, Hearnshaw H, Van Royen P, Denekens J. Patient adherence to treatment three decades of research. A comprehensive review. J Clin Pharmacol Ther. 2001;26:331-45.

34. World Health Organization. Adherence to long-term therapies: evidence for action [online]. 2003. Disponível em: URL: http://www.emro.who.int/ncd/Publications/ adherence_report.pdf 